\title{
Monitoring of automotive multistage mechanical transmissions using multi - class support vector machine
}

\section{Original Article}

\author{
Mahmoud M.A. Sayed ${ }^{1}$ and Wessam. M. Hussein ${ }^{2}$
}

${ }^{1}$ Mechanical Department, Canadian International College (CIC), ${ }^{2}$ Deptartment of Mechanical Engineering, The Military Technical College, Cairo, Egypt

\begin{abstract}
Key words:
Condition based maintenance, health monitoring system, Mechanical transmission, support Vector Machine, Vibration analysis
\end{abstract}

Corresponding Author: Mahmoud M.A. Sayed, Deptartment of Mechanical Engineering, Canadian International College (CIC) Email: mah_hsh2000@yahoo.com

\begin{abstract}
The need for semi-autonomous or autonomous operations, communication delay, short contact periods as well as the need for survival in harsh environments poses unique challenges to Automotive Mechanical Transmission Systems (AMTS). Predictive health monitoring (PHM) systems are currently gaining in popularity due to their effectiveness in providing robust information about the system condition and reducing maintenance costs. This paper presents a PHM system for monitoring different gear faults using vibration analysis and Support Vector Machine (SVM) algorithms. Experiments were conducted on a multi-stage gearbox (Automotive Mechanical Transmission Systems) under three conditions, normal, external vibrational excitation and oiling system high temperature. Multi-class SVM based on developing a model for normal and faulty states; the model used for monitoring the upcoming sensory data, and classifies them as normal or faulty ones. The model is verified through additional experimental observations. The classifier algorithm was coded in Matlab and showed a good potential in classifying different failure mechanisms.
\end{abstract}

\section{INTRODUCTION}

Monitoring the condition of the in-service mechanical transmission system is an important issue for reliability, where their components deteriorate over the time and affected much when subjected to varying loads. This led in continuous improvement of maintenance strategies from breakdown and periodic maintenance to Condition Based Maintenance (CBM) and predictive maintenance in order to sustain reliability and reducing the periodic maintenance costs. Also, in some applications there is more demanding aspect such as saving man's life other than reliability ${ }^{[1]}$. Smith $^{[2]}$, has defined the causes of transmission vibration and its transmission path, including factors such as manufacturing error, design error and gear tooth deflection, which combine to introduce a Transmission Error (TE), which is the primary source of the vibration.

Over the past decade, vibration analysis proved to be a trustworthy diagnostic technique that can provide reliable information. However, in the last 10 years researchers devoted a much effort to support CBM actions using vibration information ${ }^{[3-13]}$.

Many Researchers focused on developing multi sensors fusion algorithms to fuse vibration analysis information with other sensory data, such as Acoustic Emission (AE) and oil debris analysis to minimize false alarms that may occur in failure prediction.

Parametric methods based on mathematical modeling is used to fit measured time series waveform data to a parametric time series model, and then extract features based on this model. Two models are currently in use: the auto regressive (AR) and auto-regressive moving average (ARMA) models. The advantage of mathematical modeling based on parametric methods over the neural networks model-based method is its ability to deal with time series data directly without the need for a signal pre-processing step to extract useful features that can be modeled to represent the system. However, they can only be used to model a time series signal such as a vibration signal, and cannot be applied to combined information from several techniques (vibration and AE) such as in the case of fuzzy logic ${ }^{[14-17]}$. Also, other researchers devoted efforts to build intelligent algorithms based on vibration features including Expert systems, Artificial Neural Network (ANN's), Genetic algorithm, and fuzzy logic ${ }^{[18-29]}$.

Onsy et al. ${ }^{[30-32]}$ is devoting their efforts in developing smart CBM systems that can use one analysis technique only such as vibration or acoustic emission analysis along 
Volume (1) - Issue (1) - 2017

with intelligent algorithms to predict the onset of failures; This is to reduce costs of different sensory requirements.

The simplicity of data driven modeling approach is that there is no need for a fundamental model of the system and only data from normal operation needs to be used, which is generally available in some form for most machines. Among various clustering methods, the support vector machines (SVMs), are found to be effective in realworld applications ${ }^{[3,34]}$. In addition, the SVMs possess some useful properties for the problems of classification The simplicity of data driven modeling approach is that there is no need for a fundamental model of the system and only data from normal operation needs to be used, which is generally available in some form for most machines. Among various clustering methods, the support vector machines (SVMs), are found to be effective in real-world applications ${ }^{[33-34]}$. In addition, the SVMs possess some useful properties for the problems of classification in terms of the non linearity, efficiency of computation, and simplicity of implementation ${ }^{[35]}$. Many applications of this technique have been successfully applied in other fields of process monitoring ${ }^{[36-39]}$. He and $\mathrm{Shi}^{[40]}$ found that support vector machines produced better accuracy than artificial neural networks when applied to a pump diagnosis problem.

This paper outlines the use of the Multi-Class Support Vector Machine SVM approach, to develop a framework to monitor and test the health status of a multi stage Mechanical transmission system.

\section{SYSTEM CONFIGURATION}

An automotive mechanical transmissions gear test rig was developed for this on-going research figure 1. The rig comprises $130 \mathrm{~mm}$ centre distance gearbox and fixed on the floor using a non-vibrating platform (Fastened with rubber and bolts)

The system is driven by a $7.5 \mathrm{KW}$ variable speed 3 -phase electric motor controlled by an inverter to provide a speed variation of $1750 \mathrm{rpm}$. The system is loaded through a mechanical braking system and controlled with an AC motor inverter. The system is equipped with five sensors, two accelerometers at two different positions (input and output of the gear system), temperature sensor (immersed in the gearbox oiling system), wireless strain gauge for torque measurements (on the output shaft) and a proximity sensor for speed measurement (at the gearbox input shaft). The rig can generate a load torque on the test gears in the range of $0-200 \mathrm{Nm}$. The torque is measured using calibrated strain gauges installed on the shaft and the measured torque values are transmitted to the control program by telemetry in order to provide torque control of the loading mechanism on the mechanical transmissions. Two temperatures were measured: gearbox oil temperature and bearing temperature using RTD temperature sensors $(10 \mathrm{mv} / \mathrm{C})$. The input shaft speed and motor current were also monitored as a precaution. The test rig operating conditions were monitored and it is flexibly changed according to the required test conditions using LabVIEW's virtual instrument scalable architecture features.

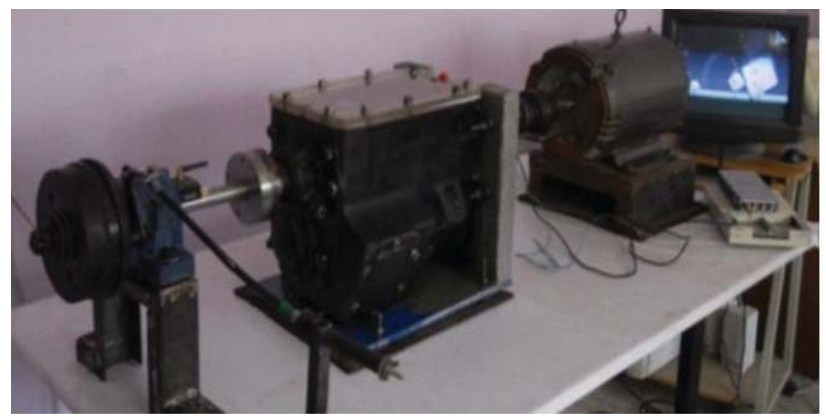

Fig. 1: Multistage gearbox system.

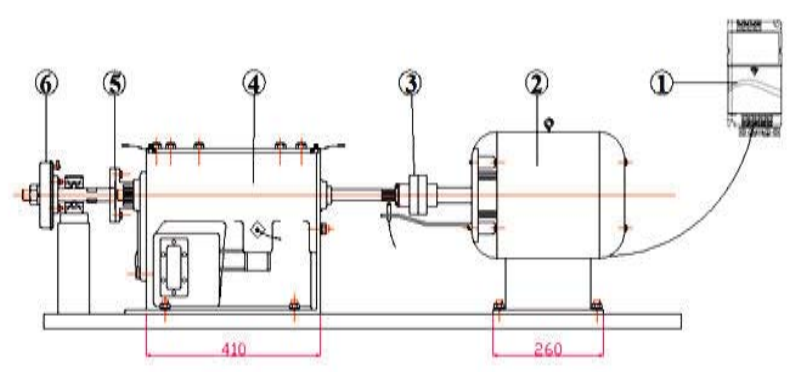

Fig. 2: Inverter, 2. Electric motor, 3. Fixable coupling, 4. 130 $\mathrm{mm}$ center distance Gearbox, 5. Mechanical coupling, 6. Loading Mechanism.

The Vibration analysis system incorporated a 24-bit NI wireless DSA data acquisition card (NI 9234 with cDAQ-9191) to acquire the vibration signal, speed and temperature. The vibration signals were acquired using two DJB Piezotronic constant current source accelerometers (model no. Acc103 -10mV/g) mounted adjacent to the tested gear bearings transversely to the gearbox casing, and a shaft speed sensor was used to acquire the shaft rotation reference. The sensors location diagram over the test rig is shown in Fig 2. The vibration signals are then acquired continuously and transmitted to the base unit using an IEEE $802.11 \mathrm{~b} / \mathrm{g}$ (Wi-Fi) wireless communication interface (frequency range 2.412-2.462 GHz). The system can send the data from a range up to $30 \mathrm{~m}$ for indoor measurements and $100 \mathrm{~m}$ for outdoor operation as long as the line of sight of the wireless signal is provided. The system can also provide Ethernet cabling measurements up to a distance of $100 \mathrm{~m}$.

\section{SUPPORT VECTOR MACHINES METHODOLOGY}

Support vector machines $(\mathrm{SVMs})^{[34,35]}$ are a group of learning machines for solving pattern recognition problems efficiently. SVMs try to find the hyperplane, which separates optimally the training patterns according to their classes (i.e. hyperplane with maximum boundary margin). This is performed by using what is commonly known in machine learning as the "kernel trick" when using SVM's. Kernel function is chosen to map the data from its original space to feature space. It can be chosen arbitrarily so as to 
best suit the data and at the same time reduce the computational burden involved with generating the mapped values by direct evaluation. "Support vectors" correspond to those points that lie along the margin or closest to it. The maximum margin between classes is found by solving a quadratic optimization problem. SVMs have a good generalization performance over traditional approaches, since their training is based on the principle of structural risk minimization (SRM) (i.e. minimizing the upper bound on the expected risk), while the training traditional approaches are based on empirical risk minimization (i.e. minimizing the number of the training error). SVMs have a high computational efficiency in terms of speed and accuracy.

They are also more preferable when dealing with high dimensional data as they are more robust than traditional approaches which may over-fit the data. However, they still have negative-aspects in terms of giving information about the system output and no physical explanation and interpretation of the process itself. The description of SVMs classification can be explained as follows:

Consider the training data $\{\mathrm{xi}, \mathrm{yi}\}$, where: $\mathrm{i}=1, \ldots, \mathrm{N}$, $y_{i} \in\{+1,-1\}$ corresponding to the class of $x i\left(y_{i}=1\right.$ for class $A, y_{i}=-1$ for class B). The principle of operation of SVMs classifier will be modified according to the type of the data samples as follows:

\section{Linearly Separable Data}

Figure 3 shows the hyper plane $\mathrm{H}$ which separates the two classes of data (separating hyper plane). This hyperplane $\mathrm{H}$ satisfies the following equality $\mathrm{b}+\mathrm{w}^{\mathrm{t}} \cdot \mathrm{x}_{\mathrm{i}}=0$

Where: $\mathrm{w}$ is a normal vector on the hyperplane, and $\mathrm{b}$ is a bias representing the distance from the origin.

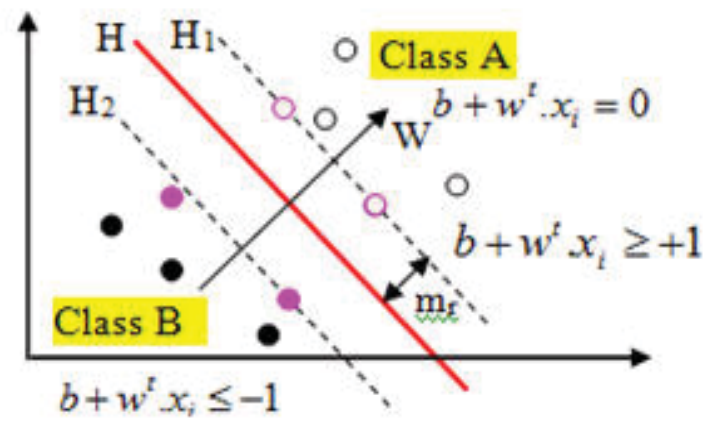

Fig. 3: Optimal separating hyperplane of SVMs for separable data.

The training data corresponding to classes $\mathrm{A}$ and $\mathrm{B}$ satisfy the following inequalities respectively

$\mathrm{b}+\mathrm{w}^{\mathrm{t}} \cdot \mathrm{x}_{\mathrm{i}} \geq 1 \quad$ and $\quad \mathrm{b}+\mathrm{w}^{\mathrm{t}} \cdot \mathrm{x}_{\mathrm{i}} \leq-1$

The two inequalities in (2) can be combined as follows $\mathrm{y}_{\mathrm{i}}\left(\mathrm{b}+\mathrm{w}^{\mathrm{t}} \cdot \mathrm{x}_{\mathrm{i}}\right) \geq 1$

The training data corresponding to classes $\mathrm{A}$ and $\mathrm{B}$ satisfy the following inequalities respectively

$\mathrm{b}+\mathrm{w}^{\mathrm{t}} \cdot \mathrm{x}_{\mathrm{i}} \geq 1 \quad$ and $\quad \mathrm{b}+\mathrm{w}^{\mathrm{t}} \cdot \mathrm{x}_{\mathrm{i}} \leq-1$
The two inequalities in (2) can be combined as follows

$$
\mathrm{y}_{\mathrm{i}}\left(\mathrm{b}+\mathrm{w}^{\mathrm{t}} \cdot \mathrm{x}_{\mathrm{i}}\right) \geq 1
$$

The equalities of (3) define hyperplanes H1, and $\mathrm{H} 2$ respectively, and any training data belongs to class A or class B and lying on $\mathrm{H} 1$ or $\mathrm{H} 2$ is called support vectors (SVs). From Fig. 4 the geometry and the separating margin of hyperplane $\mathrm{H}$ is given by

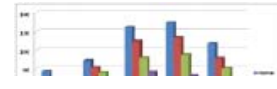

The SVMs classifier tries to find the separating hyperplane with the largest margin (optimal hyperplane). This can be formulated as follows:

Minimize $\frac{1}{2}\|w\|^{2}$

And s.t. constraints in (3)

Using the Lagrangian formulation of the problem

$$
\mathrm{L}_{p}=\frac{1}{2}\|\mathrm{w}\|^{2}-\sum_{\mathrm{i}}^{\mathrm{N}} \alpha_{\mathrm{i}} \mathrm{y}_{\mathrm{i}}\left(\mathrm{b}+\mathrm{w}^{\mathrm{t}} \cdot \mathrm{x}_{\mathrm{i}}\right)+\sum_{\mathrm{i}}^{\mathrm{N}} \alpha_{\mathrm{i}}
$$

Lp will be minimized with respect to $\mathrm{w}, \mathrm{b}$ and all the derivatives of Lp with respect to all the Lagrangian multipliers, ai will vanish. All of these multipliers are subjected to the following constraints: $\alpha i \geq 1$

The calculations can be simplified by applying Karush-Kuhn-Tucker (KKT) condition which allows applying dual formulation of the problem. This implies that the maximum of $\mathrm{Lp}$ is subjected to same constraints in (7) and acquiring that the gradient of $L p$ with respect to $\mathrm{w}$ and $\mathrm{b}$ vanishes which results in (6) and (7)

$\mathrm{w}=\sum_{\mathrm{i}} \alpha_{\mathrm{i}} \mathrm{y}_{\mathrm{i}} \mathrm{x}_{\mathrm{i}}$

$\sum_{\mathrm{i}} \alpha_{\mathrm{i}} \mathrm{y}_{\mathrm{i}}=0$

Substituting (8) and (9) in (6) results in

$$
\mathrm{L}_{\mathrm{D}}=\sum_{\mathrm{i}} \alpha_{\mathrm{i}}-\frac{1}{2} \sum_{\mathrm{i}, \mathrm{j}} \alpha_{\mathrm{i}} \alpha_{\mathrm{j}} \mathrm{y}_{\mathrm{i}} \mathrm{y}_{\mathrm{j}} \mathrm{x}_{\mathrm{i}} \mathrm{x}_{\mathrm{j}}
$$

s.t. constraints in (7) and (9). Once $\alpha$ is obtained from (10) (using a quadratic programming (QP) solver), the dimensions of the classifier $\mathrm{w}, \mathrm{b}$ are determined using (3) and (8). Substituting the obtained values of $\mathrm{w}$ and $\mathrm{b}$ in (11) allows the classification of any unknown sample.

$\mathrm{y}_{\text {unknown }}=\operatorname{sign}\left(\mathrm{b}+\mathrm{w}^{\mathrm{t}} \mathrm{x}\right.$ unknown $)$

The number of variables in (10) is the number of the training data. All the training data associated with the Lagrangian multipliers satisfying the inequality of (7) are the SVs. The number of SVs is considerably less than the number of the training data.

IV. RESULTS AND DISCUSSION

This section discusses the results of the experimental 
study, showing the application of the SVM algorithm to real stored sensory data, for normal and anomalous operation periods.

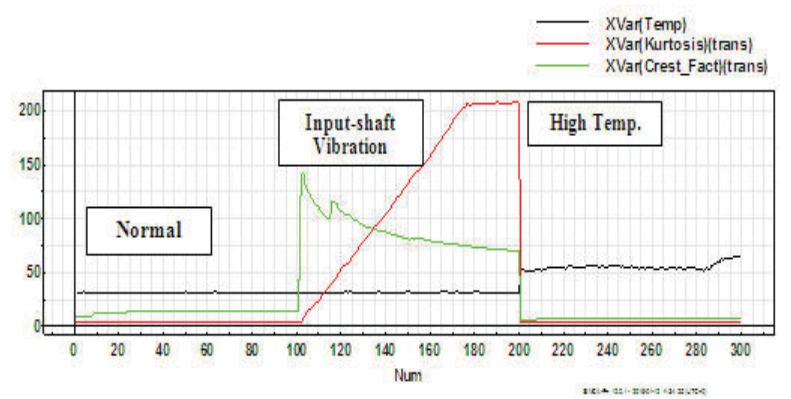

Fig. 4: Kurtosis, Crest factors and Temperature at the input gearbox shaft for 300 sampleas.

Measurements were driven through three conditions as shown in fig 4. shows Kurtosis, Crest factors and temperature of the input gearbox shaft through the different conditions. First, the system was run under normal condition (observations 1-100), then external excitations were applied at one position (input shaft: 101-200). The system was then subjected to a high temperature (201-300).

There are several evaluation schemes for selection of training and testing sets including hold-out, leave-one-out, cross-validation and bootstrap. In our article the selection is based on hold-out method. So, the 300 observations are divided into 225 observations as training set and 75 observations as testing set. The division percentage is $75 \%$ for training set to $25 \%$ for testing set.

\section{Nonlinear support vector machines results}

In this section, the generalization of the non-linear SVMs classification algorithm to the gearbox state-ofhealth data and its performance is investigated. The training technique generated in this design work is adapted to train 225 observations ( 80 normal, 145 faulty) as training set (Set-1). Subsequently, the model is tested and validated on a subset (Set-T) of the remaining 75 observations (20normal, 55 faulty) and their corresponding normalized values are directly used as the input features for SVMs. The corresponding output y1 is ( 1 for a normal condition, 2 and 3 for faulty conditions).

\section{SVM models design}

The SVM models are designed during the training process by trial and error. The training process involves different Kernel functions as well as several values of each Kernel parameters in order to obtain the SVM classifier with the best performance. The SVM and Kernel methods coded in MATLAB is used for the SVMs training and testing.

\section{Selection of Kernel function and Kernel param- eters}

The SVMs classification technique is tested for two different Kernel functions during the training process namely, the polynomial and Gaussian Radial Basis
Functions (RBF) kernel functions ${ }^{[35]}$. According to the performance of these Kernel functions, the suitability of the SVMs as an intelligent classifier is judged.

The selection of the optimum parameters for SVMs is done during the training process (Set-T). The SVM classifier with the best performance is obtained by testing different values of the Kernel parameters. These parameters are varied in the following manner; $\gamma$ is varied with values of $0.1,0.2,0.3,0.5,1,3,5$. The order of the polynomial Kernel $\mathrm{n}$ is varied in the range with steps of 2 . The penalty due to the error $\mathrm{C}$ is also varied with values of $1,10,100,500$ and 1000 . The tolerance condition for the QP solver is 0.0000001 . The performance of the two SVMs is assessed on each of these values by calculating the training percentage performance efficiencv defined bv: $\eta=\frac{\text { No. of samples correct classified }}{\text { Total No. of samples }} \times 100$

From these results, the SVM classifier with the highest training percentage performance efficiency is selected. The testing process is then performed, during which the generalization performance of the classifier is examined using testing set (Set-T) by evaluating the testing percentage performance efficiency.

\section{Training and testing the results}

Fig. 5 shows the best performance of kernel functions during the training process of the training set (Set-1).The best performance is introduced in terms of the percentage training efficiency of equation ${ }^{(12)}$, with respect to the variation of the kernel parameters $\gamma$, and $\mathrm{n}$ as well as the penalty due to the error $\mathrm{C}$. The corresponding number of SVs and the training time are also illustrated.
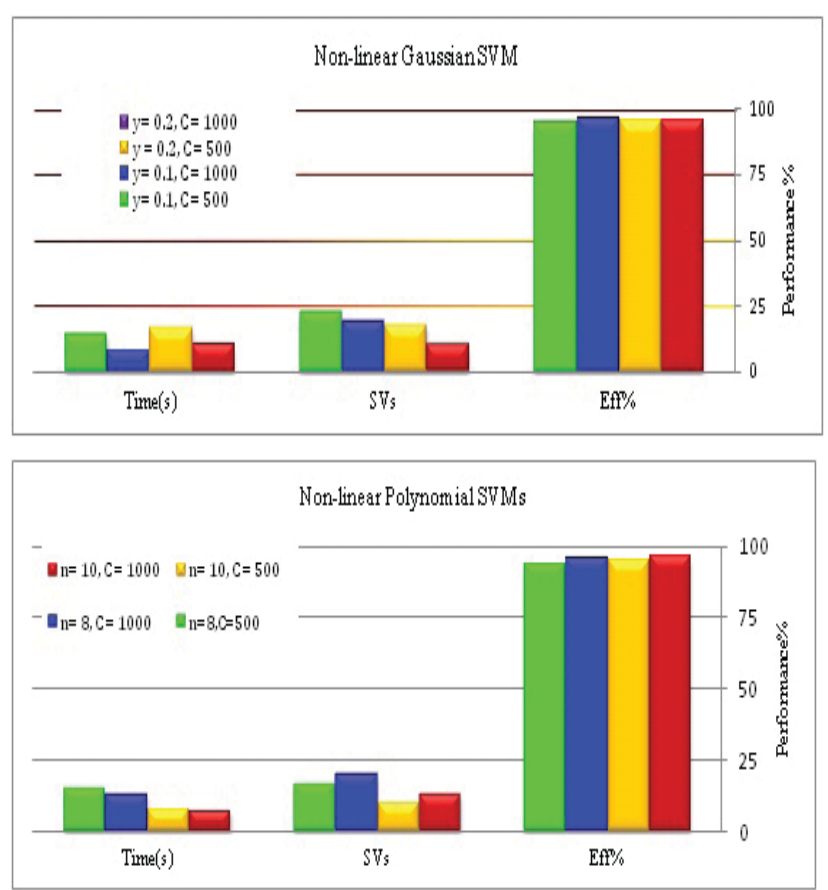

Fig. 5: Best performance obtained during training of SVMs with for normal and faulty data and for different values of Kernel parameters for Gaussian and polynomial Kernel. 
From the results illustrated in Fig. 5 the following clarification are worth noting:

1. Effect of penalty due to the error $\mathrm{C}$

a. For the Kernel functions under investigation, the best performance is obtained at high values of $\mathrm{C}=500$ and 1000. In addition, as $\mathrm{C}$ increases the training efficiency increases. 1000 .

b. The maximum training efficiency is $96.98 \%$ at $\mathrm{C}=$

2. Effect of the Kernel parameters

a. For the polynomial Kernel, as $\mathrm{n}$ increases, both of the number of SVs and the training time decrease, while the training efficiency increases. The best performance for the polynomial Kernel function is $96.13 \%$ for $\mathrm{C}=1000$, $\mathrm{n}=10$.

b. For the Gaussian Kernel, as $\gamma$ decreases, both of the number of SVs and the training efficiency increase, while the training time decreases. The best performance for the Gaussian Kernel function is $96.98 \%$ for $\mathrm{C}=1000, \gamma=0.1$.

3. Effect of the type of the kernel function

a. The best training efficiency was obtained with the Gaussian Kernel function (96.98\% during SVM training).

b. The shortest training time was obtained for the polynomial Kernel function (7.4 second during SVM training and the smallest number of SVs was obtained for both the polynomial and Gaussian Kernel function (10 SVs during SVM training).

Figures. 6 and 7 demonstrate samples of contour plots for non-linear SVM classifier using Gaussian Kernel functions. $\mathrm{C}=1000$, Gaussian, $\mathrm{KO}=2$, lambda $=1 \mathrm{e}-7$ $\mathrm{C}=1000$, Polynomial, $\mathrm{KO}=2$, lambda $=1 \mathrm{e}-7$

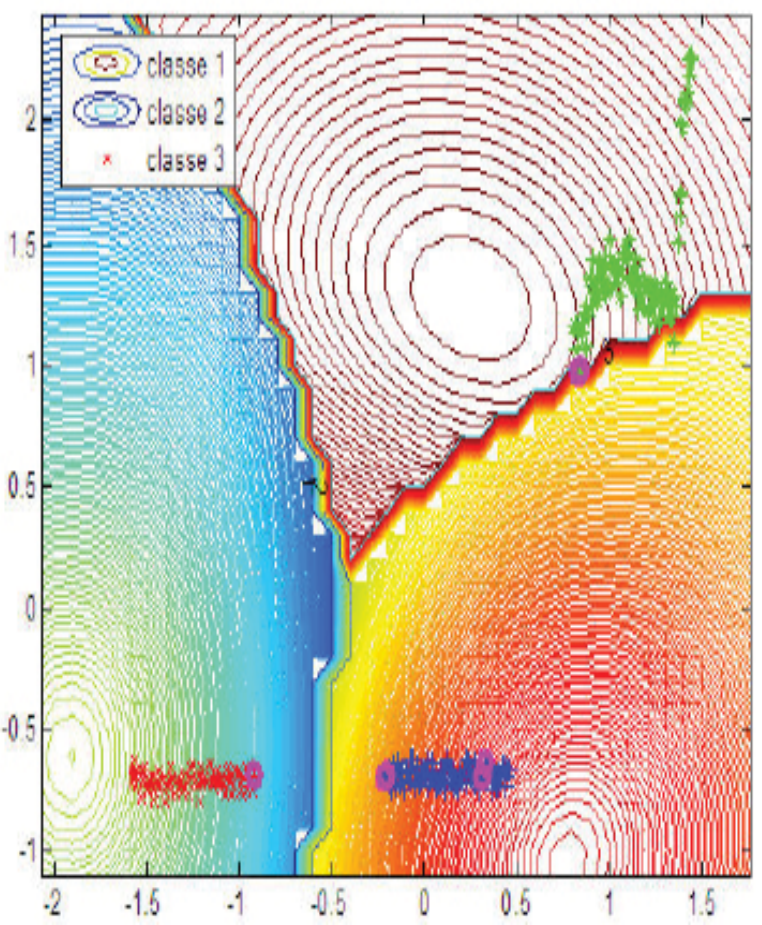

Fig. 6: Non-linear Gaussian SVMs contour plots.

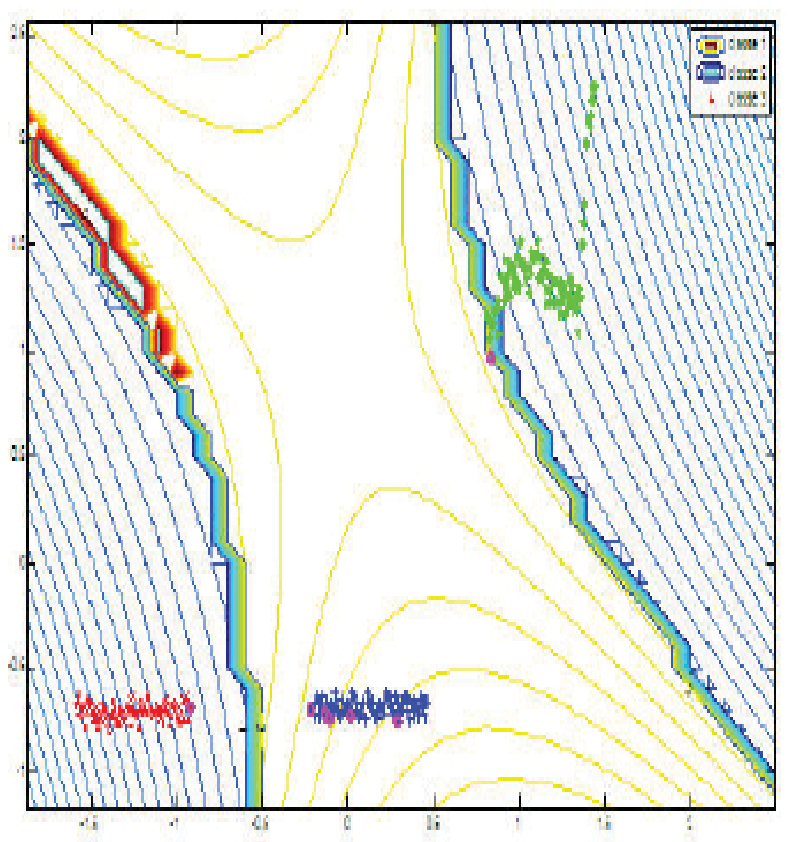

Fig. 7: Non-linear Polynomial SVMs contour plot.

\section{CONCLUSIONS}

The study has presented a wireless vibration measuring system that was able to detect different conditions of gears in automotive gearbox and clearly classify its condition using one accelerometers at input of the gear system, temperature sensor (immersed in the gearbox oiling system), wireless strain gauge for torque measurements (on the output shaft) and a proximity sensor for speed measurement (at the gearbox input shaft)for model building and testing. The study has focused on monitoring the classifying of system faults using multiclass SVM.

The system is being developed for use on $130 \mathrm{~mm}$ automotive manual transmissions, but could be adapted for other transmission or machinery systems rotating machinery.

The model was tested under different conditions including: normal condition, external vibrational excitations at one position (input shaft), high temperature and was able to successfully differentiate between them.

\section{REFERENCES}

[1] Scheffer, C. and Girdhar, P. Machinery Vibration Analysis and Predictive Maintenance, Newnes Press; ISBN 0750662751, 2004.

[2] SMITH, J.D. Gears and Their Vibration: A Basic Approach to Understanding Gear Noise, New York: M. Dekker; London: Macmillan Press, 1983

[3] ONSY, A., SHAW, B.A. \& JISHAN, Z. Monitoring the Progression of Micro- Pitting In Spur Geared Transmission Systems Using Online Health Monitoring Techniques. SAE International Journal of Aerospace. November 2014.

[4] ONSY, A., BICKER, R., SHAW, B.A., ROWLAND, C.W. \& KENT, T. Monitoring Bending Fatigue Failure in Helical Gears Using Acoustic Emission, Vibration, and On-Line Oil Debris Analysis: A Comparative Study. Proceedings of the Fifth International Conference on Condition Monitoring \& Machinery Failure Prevention Technologies, UK, Edinburgh, 2008.

[5] TAN, C.K., IRVING, P. \& MBA, D. Diagnostics and Prognostics with Acoustic Emission, Vibration and Spectrometric Oil Analysis For 
Spur Gears: A Comparative Study. Insight: Non-Destructive Testing and Condition Monitoring, 47, 478-480, 2005.

[6] AL-GHAMD, A.M. \& MBA, D. A Comparative Experimental Study on the Use of Acoustic Emission and Vibration Analysis for Bearing Defect Identification and Estimation of Defect Size. Mechanical Systems and Signal Processing, 20, 1537- 1571, 2006.

[7] G. Dalpiaz, A. Rivola and R. Rubini, Gear Fault Monitoring: Comparison of Vibration Analysis Techniques, Department of Mechanical Design Engineering University of Bologna, Italy, 2000.

[8] C. James Li, J.D. Limmer, Model-based condition index for tracking gear wear and Fatigue damage, Wear 241, 26-32, Department of Mechanical Engineering, Aeronautical Engineering and Mechanics, Rensselaer Polytechnic Institute, Troy, USA, 2000.

[9] Enayet B. Halim, Sirish L.Shah, Mingo j.Zuo and A. A. Shoukat Choudhury, Fault Detection of Gearbox from Vibration Signals using Time-Frequency Domain Averaging, 2004.

[10] Johan Wandell, Multistage Gearboxes: Vibration Based Quality Control, TRITA-AVE 2006:27 ISSN 1651-7660, Royal Institute of Technology School of Engineering Sciences Department of Aeronautical and Vehicle Engineering the Marcus Wallenberg Laboratory for Sound and Vibration Research, 2006.

[11] P.Gupta, S. B. Wadkar, Comparative Vibration and Noise Signature Analysis by Spectral, Cepstral and Wavelet Techniques, P.Gupta and S. B. Wadkar/ Advances in Vibration Engineering, 7(2) 2008 Department of Mechanical Engineering, College of Engineering, Bbartj Vidyapeeth Deemed University, India, 2008.

[12] Grzegorz Wojnar, Bogusław Lazara, Henryk Madej, Diagnostics of Power Transmissions System with Tooth Gear, Silesian University of Technology, Faculty of Transport, Department of Automotive Vehicle Construction, Poland, 2008.

[13] Matej Gasperin, ani Juricic, Pavle Boskoski, Joz ef Vizintin, Model-based Prognostics of Gear Health using stochastic dynamical models, Mechanical Systems and Signal Processing 25(2011) 537-548, Department of Systems and Control, Slovenia, 2011.

[14] ONSY, A., BICKER, R. \& SHAW, B.A. A Novel Intelligent Health Monitoring System for Gear Fatigue Failure Prediction. 23rd International Congress on Condition Monitoring and Diagnostic Engineering Management; COMADEM 2010, Japan, Nara, ISBN 978-4-88325-419-4, 2010.

[15] ONSY, A., BICKER, R. \& SHAW, B.A. Intelligent Diagnostic Health Management of Power Transmission Systems: An Experimental Validation, International Journalof COMADEM 2010, 13(2), 46-58, 2010.

[16] ONSY, A., BICKER, R., SHAW, B.A., ROWLAND, C.W. \& KENT, T. Intelligent Health Monitoring Of Power Transmission Systems: An Experimental Validation. Proceedings of the 2009 Conference of the Society for Machinery Failure Prevention Technology, USA, Dayton, Ohio, 499-518, 2009.

[17] ONSY, A., BICKER, R., SHAW, B.A., ROWLAND, C.W. \& KENT, T. Monitoring the Progression of Micro-Pitting in Helical Gears: Towards an Intelligent Health Monitoring System. Paper presented at the AEWG51 \& International Symposium on AE, USA, Memphis, Tennessee, 2008. [18] Paula J. Dempsey, Abdollah A. Afjeh, Integrating Oil Debris and Vibration Gear Damage Detection Technologies Using Fuzzy Logic, NASA/TM-2002- 211126, National Aeronautics and Space Administration Glenn Research Center, USA, 2002.

[19] Abhinav Saxena, Ashraf Saad, PhD, Evolving an Artificial Neural Network Classifier For Condition Monitoring of Rotating Mechanical Systems, Journal of Applied Soft Computing Elsevier Publishers, ISSN: 1568-4946, School of Electrical and Computer Engineering, Georgia Institute of Technology, Savannah, a 31407, USA, 2005.

[20] T.HLoutas, J.Kalaitzoglou, G.Sotiriades, V.Kostopoulos, The Combined Use of Vibration, Acoustic Emission and Oil Debris Sensor Monitored Data Coming from Rotating Machinery for the Development of a Robust Health Monitoring System, Department of Mechanical Engineering and Aeronautics, University of Patras, Patras, 2005.

[21] M.H. Sadeghi, J. Raflee, F. Arvani, and A. Harifi, A Fault Detection and Identification System for Gearboxes using Neural Networks, Center of Excellence for Mechatronics, University of Tabriz, Tabriz, Iran, 2006. [22] J. Rafiee, F. Arvani, A. Harifi, M.H. Sadeghi, Intelligent condition monitoring of a Gearbox using artificial neural network, Mechanical Systems and Signal Processing 21 (2007) 1746-1754, Department of
Mechanical Engineering, University of Tabriz,Tabriz, Iran, 2007.

[23] S.J. Loutridis, Gear failure prediction using multiscale local statistics, Engineering Structures 30 (2008) 1214-1223, Technological Educational Institute of Larissa, School of Technological Applications, Department of Electrical Engineering, Sensors an Instrumentation Laboratory, GR 41-110 Larissa, Greece, 2008.

[24] T.H. Loutas, G. Sotiriades, I. Kalaitzoglou, V. Kostopoulos, Condition monitoring of A Single Stage gearbox with artificially induced gear cracks utilizing on-line vibration and Acoustic Emission measurements, Applied Acoustics 70(2009)1148-1159, Department of Mechanical Engineering and Aeronautics, University of Patras, Patras GR-26500, Greece, 2009. [25] N. Saravanan, S. Cholairajan, K.I. Ramachandran, Vibrationbased fault diagnosis of Spur Bevel gear box using fuzzy technique, Expert Systems with Applications 36 (2009) 3119-3135, Department of Mechanical Engineering, Amrita Vishwa Vidyapeetham, Coimbatore, Tamil Nadu 641105, India, 2009.

[26] J. Rafiee, P.W.Tse, (2009) Use of autocorrelation of wavelet coefficients for fault diagnosis, Mechanical Systems and Signal Processing 23- 1554-1572, Department of Mechanical, Aerospace and Nuclear Engineering, Jonsson Engineering Center, Troy, USA, 2009.

[27] J. Rafiee, M.A. Rafiee, N. Prause , P.W. Tse, Application of Daubechies 44 in Machine Fault Diagnostics, Dept. of Mechanical, Aerospace \& Nuclear Engineering, Rensselaer Polytechnic Institute, NY, USA, 2010

[28] Piotr Czech, .Boguslaw Lazar, Henryk Madej, Grzegorz Wojnar, Classification Of Tooth Gear Wheel Faults of Gearbox Working In the Circulating Power Test Rig By Multilayer Perceptron and Continuous Wavelet Transform, ACTA Technica Corviniensis - Bulletin of Engineering ISSN: 2067-3809, Faculty of Transport, Silesian University of Technology, Gliwice, POLAND, 2010.

[29] Hongyu Yang, Joseph Mathew and Lin Ma, Vibration Feature Extraction Techniques For Fault Diagnosis of Rotating Machinery-A Literature Survey, Asia- Pacific Vibration Conference, 12-14 November 2003, Gold Coast, Australia, School of Mechanical Manufacturing and Medical Engineering, Australia, 2003.

[30] ONSY, A., BICKER, R. \& SHAW, B.A. Predictive Health Monitoring of Gear Surface Fatigue Failure Using Model-based Parametric Method Algorithms; An Experimental Validation. SAE International Journal of Aerospace. September 2013 Issue, 2013.

[31] ABELAZEZ, W.,ONSY, A.\& SHERIF, I. Health Monitoring of Electro-Pneumatic Controlled Systems using Multivariate Latent Methods: An Experimental Validation, SAE International Journal of Materials and Manufacturing Issue, 2013.

[32] ONSY, A., BICKER, R., SHAW, B. A. \& FOUAD, M. Application of Image Registration Techniques in Monitoring the Progression of Surface Fatigue Failures in Geared Transmission Systems. The AeroConf IEEE Aerospace Conference, USA, Big Sky, 2012.

[33] C. S. Tyagi, "A comparative study of SVM classifiers and artificial neural networks application for rolling element bearing fault diagnosis using wavelet transform preprocessing,"in Proc. World Academy of Science, Engineering and Technology, pp. 319-327, 2008.

[34] Vapnik, V. "Support-vector networks". Machine Learning ,20 (3): 273-297, 1995.

[35] V. Vapnik, The Nature of Statistical Learning Theory, $2^{\text {nd }}$ ed., New York: Springer, Nov. 2000.

[36] Dingguo Lu and Wei QiaoAdaptive," Feature Extraction and SVM Classification for Real-Time Fault Diagnosis of Drivetrain Gearboxes," Energy Conversion Congress and Exposition (ECCE), IEEE, 3934 3940, 2013.

[37] T Praveenkumar, M Saimurugan, $\mathrm{P}$ Krishnakumar, K I Ramachandran,"Fault diagnosis of automobile gearbox based on machine learning techniques," 12th GLOBAL CONGRESS ON MANUFACTURING AND MANAGEMENT, GCMM 2014.

[38] Lixin Gao, Zhiqiang Ren, Wenliang Tang, Huaqing Wang, and Peng Chen,"Intelligent Gearbox Diagnosis Methods Based on SVM, Wavelet Lifting and RBR,"Sensors, ISSN 1424-8220, 10, 4602-4621, 2010.

[39] Pedro Santos, Luisa F. Villa , Aníbal Reñones , Andres Bustillo and Jesús Maudes,"An SVM-Based Solution for Fault Detection in Wind Turbines," Sensors, ISSN 1424-8220, 15, 5627-5648, 2015.

[40] He, F., and Shi, W. WPT-SVMs based approach for fault detection of valves in reciprocating pumps. In Proceedings of the American Control Conference, 2002. 